\title{
Plasmonic Metalattices: A Correlated Monochromated Electron Energy Loss Study and Theoretical Calculations
}

Parivash Moradifar ${ }^{1}$, Lei Kang ${ }^{2}$, Pratibha Mahale 3 , Yunzhi Liu ${ }^{3}$, Nabila N. Nova ${ }^{3}$, Andrew Glaid ${ }^{3}$, Thomas E. Mallouk ${ }^{3}$, John Badding ${ }^{3,4}$, Douglas Werner ${ }^{2}$ and Nasim Alem ${ }^{1}$

1. Department of Materials Science and Engineering, Materials Research Institute, The Pennsylvania State University, University Park, USA.

2. Department of Electrical Engineering, The Pennsylvania State University, University Park, USA.

3. Department of Chemistry, The Pennsylvania State University, University Park, USA.

4. Department of Physics, Department of Chemistry, Department of Materials Science and Engineering, The Pennsylvania State University, University Park, USA.

Various noble metals (gold and silver) based nanostructures have been extensively studied for their wellknown plasmonic responses [1,2]. However, there are strong motivations in exploring new plasmonic structures that exhibit lower losses and more functionally diverse properties. Metamaterials have been proposed because of their highly tunable optical properties through engineering the internal structures that may not exist in nature [3].

Metalattices as a subgroup of metamaterials, are nanostructured 3D ordered hybrid materials on the range of sub $100 \mathrm{~nm}$ (sub wave-length scale) [1-3]. A metalattice structure is comprised of dielectric silica nanospheres and meta-atoms linked through thin interconnected channels named meta-bonds. A void-free infiltration of these 3D ordered frameworks with semiconducting materials and/or metals can provide a versatile and tunable platform as integrated plasmonic interconnects for large optical confinement and long propagation distance applications. Recently, chemical fluid deposition from supercritical carbon dioxide, has been shown as a robust and cost-effective approach to achieve void-free infiltration of such hybrid systems $[4,5]$.

In this study, monochromated electron-energy loss spectroscopy (EELS) in conjunction with aberration corrected scanning transmission electron microscopy (STEM) and x-ray energy dispersive spectroscopy (XEDS) was used to undertake a detailed study of nanoscale optical response variations in metalattices. This has been done by indirect mapping of the electromagnetic hotspots associated with the localized surface plasmon resonances (SPRs) at the nanoscale level on silver filled metalattices as a novel plasmonic structure for a wide spectral range across visible and UV regime and highly doped semiconductors (p-Si) filled metalattices as a potential tunable plasmonic material in the near-infrared regime. In addition, SiGe core-shell metalattices with etched cavities are studied to explore the effect of periodic cavities on optical response variations in this structure. This work will also combine CST calculations to further elaborate the experimental EELS measurements.

Figure 1a and b, show HAADF-STEM image of a cross-section Ag filled metalattices with an HAADFSTEM image from the marked ROI respectively. Figure 1c shows the experimental low-loss EEL spectrum of marked regions (green ROI) in Figure 1b, indicating 5 different peaks corresponding to a dipolar mode at below $1 \mathrm{eV}$ (peak\#1) followed with a quadrupolar mode (peak \#2), higher multipolar modes $($ peak\#3,4) and a bulk plasmon mode (peak\#5) ${ }^{[2]}$. 2D EELS intensity maps of surface and bulk plasmon modes at indicated energies along with CST simulation are presented in Figure 1d indicating highly confined and locally enhanced electromagnetic field induced by surface plasmon excitations. As presented in Figure1d, 
meta-bonds are providing a greater field enhancement effect because they have larger energy confinement than meta-atoms. Figure 1e shows a low magnification HAADF-STEM image of 60nm Si-Ge core-shell metalattice with periodic etched cavities. XEDS compositional maps of $\mathrm{Si}$ and $\mathrm{Ge}$ along with the corresponding HAADF-STEM are presented in Figure 1f-k.

Plasmonic metalattice as a periodic and long-range interconnected structure is a novel route to make highly tunable and cost-efficient plasmonic materials with enhanced photonic properties. Controlling the size/geometry, the dopant level and a template with drastically different dielectric function from the infiltrated species provide a significant control over ultra-local modification of surface plasmon properties in plasmonic metalattices leading to a more efficient electromagnetic energy storage, enhanced biological and chemical sensing and optical data storage [6].

\section{References:}

[1] AL Koh et al., ACS Nano 3 (2009), p. 3015.

[2] O Nicoletti et al., Optics Express 19 (2011), p. 15371.

[3] JJ Cha et al., Nano Lett. 13 (2013), p. 5913.

[4] Y Liu et al., Nano Lett. 1 (2018), p. 546.

[5] P Moradifar et al., Microscopy and Microanalysis 24 (S1), p. 432.

[6] This work was funded by the Penn State MRSEC, Center for Nanoscale Science, under the award NSF DMR-1420620.
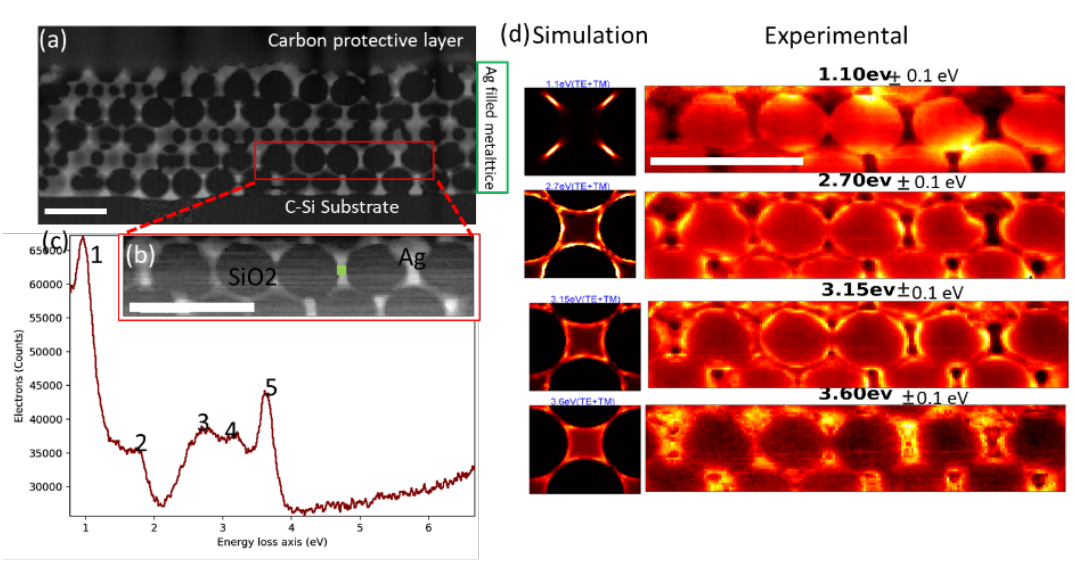

(e)

Si-Ge core-shell metalattice

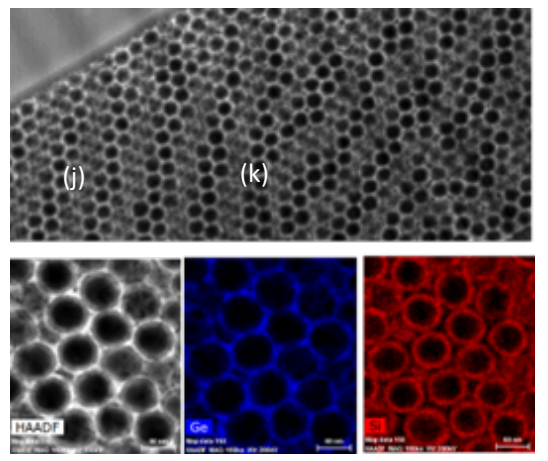

Figure 1. (a) Low magnification HAADF-STEM image of 100nm Ag metalattice (b) HAADF-STEM signal from the marked ROI in (a) (c) low-loss EELS spectrum acquired from marked regions on (b) with 5 different peaks corresponding to a dipolar mode at below $1 \mathrm{eV}$ (peak \#1) followed with a quadrupolar mode (peak \#2), higher multipolar modes (peak\#3,4) and a bulk plasmon mode (peak\#5) (d) 2D EELS intensity maps of the surface and bulk plasmon modes at indicated energies along with CST simulation in $100 \mathrm{~nm}$ Ag metalattice (e) HAADF-STEM image of 60nm Si-Ge core-shell metalattice (f-k)Si and Ge XEDS compositional maps along with the corresponding HAADF-STEM. Scale bar=200nm 